A N N A L E S Annales de Bretagne et des Pays de l'Ouest

\title{
Boquen entre utopie et révolution 1965-1976
}

\section{Frédéric Le Moigne}

\section{OpenEdition}

Journals

Édition électronique

URL : http://journals.openedition.org/abpo/3260

DOI : $10.4000 /$ abpo.3260

ISBN : 978-2-7535-5040-7

ISSN : 2108-6443

Éditeur

Presses universitaires de Rennes

Édition imprimée

Date de publication : 22 avril 2016

Pagination : 221-223

ISBN : 978-2-7535-5038-4

ISSN : 0399-0826

Référence électronique

Frédéric Le Moigne, "Boquen entre utopie et révolution 1965-1976». Annales de Bretagne et des Pays de

I'Ouest [En ligne], 123-1 | 2016, mis en ligne le 22 avril 2016, consulté le 23 septembre 2020. URL:

http://journals.openedition.org/abpo/3260 ; DOI : https://doi.org/10.4000/abpo.3260 
mères d'A.D. Dans une très brève introduction, elle notait que certains d'entre eux se retrouvaient dans le folklore paysan mais que, et cela est intéressant au plus haut point, le passage de bouche éduquée en oreille instruite les avait transformés et leur avait fait perdre " une partie de leur saveur primitive ". En quelque sorte du Pourrat, mais ici spontané et inconscient. Les vingt contes publiés appartiennent tous à la tradition gallèse et bretonnante, dans la mouvance d'un Luzel ou d'un Sébillot, et se rattachent tous à la galaxie des contes français ou occidentaux.

Le livre est utilement complété par plusieurs annexes, la liste de titres, les références aux grands catalogues de chansons et de contes (Coirault et Laforte pour les chansons, Delarue-Tenèze et Aarne-Thompson pour les contes), enfin, un glossaire des termes gallos. Un tel ouvrage fait plaisir et, on l'espère, pas seulement au petit cercle des spécialistes, folkloristes et historiens.

Jean-François TANGUY

LEBEL, Béatrice, Boquen entre utopie et révolution 1965-1976, Rennes, PUR, coll. " Histoire ", préface d'Étienne Fouilloux, postface de Bernard Besret, 2015, 394 p.

Comme Plogoff, Boquen est devenu au tournant des années 1960 et 1970 un lieu commun de contestation bretonne. Nulle intervention jugée abusive de l'État en périphérie bretonne ni affrontement avec des compagnies de CRS dans ce cas, mais bien la cristallisation d'une " crise dans la crise " qui touche l'Europe catholique de l'Ouest à la sortie du concile Vatican II. Le cadre est parfaitement romantique : une vieille abbaye cistercienne du Mené (act. Côtes-d'Armor) restaurée par dom Alexis Presse (1883-1965) depuis 1936 pour y accueillir une - fragile - communauté monastique; un jeune héritier prometteur ensuite, Bernard Besret, fils spirituel de dom Presse et lui-même prieur de Boquen dès l'âge de trente ans, après une adolescence pourtant passée loin des bancs de l'Église. Aussi magnétique (Yves Congar parle de " magnétisme humain " pour cet admirateur d'Aldous Huxley...) et charismatique que son prédécesseur, il attire à lui, par sa rénovation intense de la liturgie, puis par ses audaces sacramentelles, son ouverture aux divorcés et aux prêtres mariés ou son souffle contestataire, quantité de " chrétiens en recherche " et de catholiques de gauche. Boquen et son christianisme " critique, lyrique et politique " devient un haut lieu, sinon le haut lieu, de la jeunesse contestataire de l'Église de France sans réussir à suivre totalement et à capter l'énergie politique du mouvement de même qu'à suivre sa géographie complexe et morcelée entre projections régionalistes et réflexes parisianistes. L'expérience dure dix ans, entre 1965 et 1975. Reste le souvenir de la vitalité mais aussi des impasses d'une communauté nouvelle reliée à une "Communion " plus vaste mais en conflit avec les soutiens locaux et dont la plasticité succède à une structure elle-même en recherche sous dom Alexis. Celle-ci se heurte, dans un très long feuilleton, à l'autorité des pères : l'ordre cistercien d'une part et les évêques d'autre part, dont les champs d'autorité bien que communs sont loin de se confondre, ce dont use d'ailleurs parfaitement Bernard Besret, à son aise sur le terrain juridique.

L'ouvrage de Béatrice Lebel est dès le départ la chronique d'un échec annoncé, celui d'un lieu plus rêvé et intellectualisé, de l'intérieur comme de l'extérieur, que réel : une utopie qui n'a pas connu de véritable révolution, sinon celle de l'implosion de la communauté par division progressive de ses membres et surtout par l'effacement et la lassitude de son leader. De cela il ne resterait peut-être plus grandchose sans la trace de ses riches archives laissées par Bernard Besret au Centre de 
Recherches Bretonnes et Celtiques de Brest et, désormais, cette synthèse définitive. Ce que l'historienne nomme son " hygiène positiviste " est en fait un long et exigeant compagnonnage avec un fonds qu'elle a elle-même patiemment classé et maitrisé, bonifié par des entretiens, éprouvé par la discussion continue avec son directeur de thèse, Yvon Tranvouez, lui-même très impliqué dans ce travail de compréhension de la crise catholique. La justesse de ce dispositif qui est en fait sa force dans l'écriture est d'avoir ensuite laissé l'histoire s'inventer au jour le jour en respectant dans sa chronique la liberté et le rythme des acteurs, en pointant sans le jugement de trop leurs contradictions et leurs apports. Le résultat est une radiographie complète de la crise de Boquen ou, écrit autrement, l'œuvre impeccable d'une apicultrice sachant écrire et non simplement décrire sa ruche.

Bien sûr, cela n'enlève pas un certain pessimisme, qui transparaît dans l'habile montage photographique qui accompagne cet ouvrage. Foin de nostalgie soixantehuitarde, le noir et blanc n'est pas là pour reproduire la couleur des pull-overs de Bernard Besret et préfère montrer un "trou ", nom de la salle des discussions de Boquen! La dépression, la tension mentale des protagonistes sont évoquées avec pudeur. Le degré conscient de manipulation de la part du principal protagoniste de cette aventure peut être discuté à l'infini. On se contentera de signaler que dans sa postface, Bernard Besret fait un habile pied de nez à l'histoire - positiviste puisqu'après avoir confié ses archives, il préfère parler de ces événements de papier qui n'ont laissé aucune trace dans son souvenir pour mieux évoquer des faits qui n'ont laissé aucune trace dans les archives... Au passage, il évoque ses relations finalement amicales avec les évêques pour mieux souligner le fait que le cardinal Gouyon, archevêque de Rennes, l'a traité d'hérétique (soit une forme d'inquisition à la mode de la courtoisie conciliaire).

Béatrice Lebel a donc bien raison de rester sur ses gardes en évoquant un rapport au passé " étonnamment apaisé " chez Bernard Besret, elle qui sort de la tension d'une correspondance hautement exigeante et parfois éprouvante dans son dévoilement des êtres. Ce n'est donc pas se tromper d'enjeu que d'aller sur le terrain du jugement moral dans ce compte rendu historien tant ce Boquen apparaît avec évidence comme le canevas d'une pièce. S'il s'agit de théâtre de l'intime, la préface d'Étienne Fouilloux nous invite également à ne pas minimiser le poids historique de cette affaire. Pourquoi? La restauration voulue par Jean-Paul II a recouvert " d'un voile pudique " la mémoire de la "crise catholique " et entraîné une déconsidération des engagements des uns et des autres. En termes de modalités de mobilisation catholique que Béatrice Lebel lit indirectement avec la grille de Jean Séguy (sociologie de la contestation) ou celle d'André Rousseau, on se trouve en effet face à des pratiques de débat, de figures d'influence (François Biot, Françoise Vandermeersch), de jonctions médiatiques et de réseaux (ceux de Vie Nouvelle ou des amis de Témoignage Chrétien), de manifestations (Boquen 1969, Bourges 1970, Rennes 1972) qui manquent d'héritiers et d'héritage, en dépit du fort investissement historiographique. À l'inverse, on perçoit très bien à la lecture de sa correspondance comment Bernard Besret est un parfait professionnel de la contestation moderne. "Tu as extraordinairement le sens de l'événement ", lui écrit Olivier du Roy, alors abbé de Maredsous. Bernard Besret sait parfaitement jouer de la petite phrase pour les plantes carnivores que sont les journaux. Sa modernité n'est absolument pas périmée et tendrait même à faire penser - c'est la thèse de certains actuellement (Gaël Brustier, Le Mai 68 conservateur, Cerf, 2014) - que les mobilisations conservatrices récentes restent marquées à leur manière par l'esprit de 68. Le relais médiatique en fait foi.

Cela conduit à s'interroger sur la valeur des provocations de Bernard Besret l'année sabbatique pour les prêtres et religieux (août 1969) ou l'eucharistie - « apé- 
ritif " (décembre 1969). La lecture est double : on peut y voir une forme d'humour, trait de l'esprit contestataire de 68 . Le rire serait la transgression ultime mettant le religieux face à ses dernières contradictions; ce qui explique la levée de boucliers des autorités qui " tombent dans le panneau " recherché par les provocateurs de la jeunesse. Toute la question est cependant de savoir s'il s'agissait véritablement d'humour de la part d'acteurs aussi engagés et dont les introspections n'étaient pas forcément gages de mise à distance.

Quarante ans après les faits, Boquen qui reste un enjeu de mémoire dont témoigne la fermeture des archives romaines et de certaines archives diocésaines, plus comptables des blessures de l'Institution que soucieuses d'appliquer la doctrine Decourtray issue de l'affaire Touvier, est donc devenu un très bel objet d'histoire et de compréhension de l'intérieur de la contestation catholique post-conciliaire.

Frédéric LE MOIGNE

TOINARD, Roger, Du trou noir à l'embellie ou l'histoire de l'émigration costarmoricaine de la Révolution à nos jours, sl, 2012, 438 p.

Cet ouvrage autoédité constitue une sorte de bilan des réflexions et recherches personnelles engagées il y a environ un demi-siècle par le géographe Roger Toinard sur l'histoire socioéconomique du département des Côtes-du-Nord (puis Côtesd'Armor), département dans lequel R. Toinard a fait une partie de ses études, exercé son métier d'enseignant et vit encore aujourd'hui. Il est centré sur la question de l'émigration costarmoricaine à l'époque contemporaine, un choix doublement justifié, et par l'importance singulière que le phénomène de l'émigration a revêtue dans les Côtes-du-Nord au cours des deux derniers siècles, et par le fait que ce phénomène n'avait cependant jamais fait l'objet d'un essai de synthèse. Cela dit, désireux d'expliquer autant que possible les dynamiques migratoires et même, plus largement, démographiques placées au cœur de sa réflexion, l'auteur consacre régulièrement des développements à l'histoire économique et sociale, voire culturelle des Côtes-du-Nord, si bien qu'à certains égards il en vient à proposer un ouvrage de portée assez générale sur l'histoire contemporaine du département. Il faut préciser que Du trou noir à l'embellie est destiné à un " large public " (p. 6) et comporte à ce titre quelques mises au point inutiles pour le chercheur, et qu'il possède, d'autre part, une ambition qu'on pourrait qualifier de politique, au sens où il prétend aider les Costarmoricains à penser plus efficacement l'avenir de leur territoire. Le propos est fondé à la fois sur la littérature scientifique existante, récente ou ancienne, ainsi que sur deux sources à proprement parler : les recensements de population, dont les enseignements constituent la véritable armature de la démonstration, et des témoignages de migrants originaires des Côtes-du-Nord recueillis à l'occasion de deux campagnes de collecte, dans les années 1960 et 2000.

Le livre est structuré chronologiquement. Il se compose de trois parties correspondant, à peu près, aux trois grands cycles de hausse et de baisse de la population costarmoricaine. La première s'étend du tout début du XIX'e siècle à 1866 et couvre le premier de ces cycles, qui voit passer le nombre d'habitants du département de 504000 à 641000 - si l'on arrondit au millier le plus proche. Croissance forte, donc, surtout dans le premier tiers du siècle, mais résultant de la forte vitalité naturelle de la population et dissimulant en fait une précoce et déjà forte émigration : R. Toinard chiffre à 100000 environ le nombre de départs survenus entre 1801 et 1866 . Ces derniers sont liés au premier chef à l'effondrement de l'activité toilière et touchent donc 